\title{
Efficacy and Safety of Lenalidomide in HIV- associated Cryptococcal Meningitis (HIV-CM) Patients with Persistent Intracranial Inflammation】 An Open-label, Single-arm Prospective Cohort Study
}

\section{Ran Tao}

National Clinical Research Center for Infectious Diseases, Zhejiang University

\section{Zhikai Wan}

National Clinical Research Center for Infectious Diseases, Zhejiang University

Jiangjin Hui

National Clinical Research Center for Infectious Diseases, Zhejiang University

Xiang Liu

National Clinical Research Center for Infectious Diseases, Zhejiang University

\section{Xiaorong Peng}

National Clinical Research Center for Infectious Diseases, Zhejiang University

\section{Yongzheng Guo}

National Clinical Research Center for Infectious Diseases, Zhejiang University

Xueling Zhu

National Clinical Research Center for Infectious Diseases, Zhejiang University

\section{Ying Hang}

National Clinical Research Center for Infectious Diseases, Zhejiang University

Biao Zhu ( $\sim$ zhubiao1207@zju.edu.cn )

National Clinical Research Center for Infectious Diseases, Zhejiang University

\section{Research Article}

Keywords: HIV-associated cryptococcal meningitis, lenalidomide, immunomodulatory drug, persistent intracranial inflammation,

Posted Date: February 7th, 2022

DOI: https://doi.org/10.21203/rs.3.rs-1320521/v1

License: (a) This work is licensed under a Creative Commons Attribution 4.0 International License. Read Full License 


\section{Abstract}

Background Several HIV-associated cryptococcal meningitis (HIV-CM) patients were found to have persistent intracranial inflammation despite negative Cerebrospinal fluid (CSF) fungal cultures after being optimally treated for $\mathrm{CM}$, which could be devastating for the central nervous system. However, there is no definitive treatment strategy for this condition.

Method We identified 14 HIV-CM patients with persistent intracranial inflammation and conducted a 24 weeks, prospective cohort study. All participants received oral lenalidomide $25 \mathrm{mg}$ on days 1 to 21 of a 28-day cycle. The follow-up lasted for 24 weeks with visits performed the baseline, week 4,8,12,24. The primary endpoint was the efficacy of lenalidomide therapy which was determined by the clinical manifestations, the changes in routine CSF parameters, and radiological findings within 24 weeks posttreatment. In addition, an exploratory analysis was made on the changes of CSF cytokine. Safety and efficacy analysis was performed in patients who received at least one dose of lenalidomide.

Results Of the 14 participants, 11 patients completed the 24 weeks of follow-up. it was observed that rapid clinical remission followed lenalidomide therapy, clinical presentation such as fever, headache, and cognitive impairment was fully recovered at week 4 and remained stable during follow-up. And a significant reduction of CSF white blood cell (WBC) count occurred at week 4 [Median count 3.00×10 6/L (IQR 0-45.00 P=0.009)] from baseline [35×10 6 /L (IQR 4.50 to 90.00)]. Median CSF protein concentration decreased from $1.39 \mathrm{~g} / \mathrm{L}$ (IQR 0.74-3.23) at baseline to $0.91 \mathrm{~g} / \mathrm{L}$ (IQR 0.63-1.41) at week 4 (P=0.004). CSF WBC count, CSF protein remained stable and approached the normal range through week 24 . And after the 24-week lenalidomide therapy, CSF IgG levels were also decreased, though not statistically significant. Brain MRI demonstrated that the multiple lesions were also absorbed post-therapy. In addition, it was observed that TNF-a G-CSF, IL-6, IL-17A decreased significantly during the 24-week follow-up. 2(14.3\%) patients had a mild skin rash, which resolved spontaneously without drug interruption. No study drugrelated serious adverse events were observed and no patient withdrew from therapy because of unacceptable toxicity.

Conclusion Our study found that lenalidomide can significantly improve persistent intracranial inflammation of HIV-CM patients and have well tolerance and without notable side effects.

\section{Introduction}

Cryptococcal meningitis (CM) is a form of invasive infectious disease caused by the fungus Cryptococcus neoformans, which primarily occur inimmunocompromised hosts such as people living with human immunodeficiency virus (PLWH)[1]. CM is a subacute meningoencephalitis, but its pathogenicity increases in PLWH, eventually maybe life-threatening [2]. It is reported that CM is a major cause of mortality in people with advanced HIV infection, especially in Africa and southeast Asia[3]. And the annual fatalities from HIV-associated cryptococcal meningitis (HIV-CM) are about 180,000 deaths worldwide, accounting for $15 \%$ of HIV-related mortality [4]. Recently, the mortality of patients with HIV-CM 
has significantly decreased by active antifungal therapy, aggressive management of raised intracranial pressure, and timely antiretroviral therapy (ART)initiation [5]. In clinical practice, it was observed that after long-term effective antifungal treatment (amphotericin $B$, flucytosine, fluconazole) and ART, the cerebrospinal fluid (CSF) cryptococcus culture has become negative andtheplasma HIV-RNA undetectable in the patients. However, some of them continued to haveabnormal CSF parameters and clinical symptoms persist or even worse[6]. Their clinical features are similar to encephalitis syndrome and the clinicalmanifestations mainly are fever, headache, seizures, and even cognitive dysfunction. At the same time, the radiological examination shows aberrant leptomeningeal enhancement, new parenchymal brain lesions, white matter lesions,etc[7, 8].

Previous studies showed that this phenomenon occurs because of persistent intracranial inflammation mediated brain injury[9].Despite the ART, the residual low levels of viral replication from HIV latent reservoirs and the subsequent antiviral immune response may be the cause of sustained immune activation in the central nervous system (CNS)[10].Subsequently, the positive feedback loop of immunemediated tissue damage and repair processes can lead to persistent chronic CNS inflammation [9]. On the one hand, chronic CNS inflammation caninduce neuronal insults, leading to cognitive dysfunction[11],and on another hand, continuous intracranial inflammation can lead to increased intracranial pressure and brain oedema, thereby increasing the risk of seizures and brain herniation [12].Therefore, safe and effective interventions are urgently needed for these patients to manage persistent intracranial inflammation to improve their clinical outcomes.Mechanismstudies suggest thatthepersistent intracranial inflammationare characterized by high levels of CSF T helper1 (Th1)associated proinflammatory cytokines suchasinterferon- $\gamma$ (IFN- $\gamma$ ), tumor necrosis factor- $a(T N F-a)$, granulocyte colony-stimulating factor (G-CSF) and interleukin(IL)-6[13, 14]. Thus, anti-inflammatory or immunomodulatory methods have become the primarytherapy options for persistent intracranial inflammation.Several case reports documented neurological improvement after the use of immunosuppressive drug corticosteroids, hydroxychloroquine thalidomide and adalimumab[15]. However, due to the lack of prospective clinical trial data and the small number of patients treated, it is not yet sufficient to judge the efficacy and safety of the above immune modulators[16].

Lenalidomide, a TNF-aantagonist has been shown to exert immunomodulatory and anti-inflammatory effects and is a widely-used and effective antitumor agent[17, 18]. As an analogue of thalidomide, lenalidomide is more potent in immunomodulation and with fewer side effects [19]. In animal models, lenalidomide has been shown to cross the blood-brain barrier and is attempted as an adjuvant treatment for tuberculous meningitis[20]. Due to its low neurotoxicity, lenalidomide has been tried for meningeal myeloma and central nervous system lymphoma[21, 22]. We conducted a prospective interventional clinical trial to determine whether the lenalidomide improved clinical outcomes of HIV-CM patients with persistent intracranial inflammation. We regular assessedthe clinical features ofthe enrolled individual including clinical presentations, laboratory features, and imaging characteristics, and we evaluated clinical outcomes after 6 months of follow-up.In addition, the cytokine concentrationsin the CSF of participants were measured and compared pre and post-lenalidomide therapy 


\section{Methods}

\section{Study design and participants}

This is an open-label, single-arm cohort trial conducted in National Clinical Research Center for Infectious Diseases Zhejiang, China. Primary inclusion criteria for this study were HIV-1 infected individuals (see Supplementary Material forthe definition of primary HIV infection) with proven successful induction therapy for $\mathrm{CM}$, a positive diagnosis of inflammation in the central nervous system $₫ \mathrm{CSF}$ protein is higher than $0.45 \mathrm{~g} / \mathrm{L}$ or CSF white blood cell (WBC) countis greater than 8/uLor cranial radiological examination revealed abnormal signal lesions such as inflammation, edema and so on $\llbracket$. Patients with severe underlying diseases of the heart, brain, liver, and kidney, absolute neutrophil count at 1000 cells per $\mu \mathrm{L}$ or less, platelet count less than $75000 / \mathrm{ul}$, a known hypersensitivity or contraindication to lenalidomide, pregnancy, or breast-feeding were excluded (seeSupplementary Material for full criteria). The trial protocol and other documents were approved by the Institutional Ethics Committee in First Affiliated Hospital of Zhejiang University in accordance with the Helsinki Declaration. Informed consent was obtained from all patients in the trial. This study is registered with China Clinical Trial Registry website (www.chictr.org/cn/, Number: ChiCTR1900023184).

\section{Procedures}

This is a single-arm prospective study, all participants received oral lenalidomide $25 \mathrm{mg}$ on days 1 to 21 of a 28-day cycle. Treatment andobservation lasted six months.Before enrollment, eligible individuals participated in a screening visit, in addition to the laboratory examination, a comprehensive medical history evaluation was also performed. And we scheduled all participants to receive a series of 5 visits over 24 weeks (at baseline, week 4, week 8, week 12, week 24). At all visits, we assessed the clinical symptoms and adverse reactions of all eligible individuals and performed blood tests including full blood counts, liver and kidney function tests, coagulation function, C-reactive protein, CD4+ T-lymphocytes T cell counts, and HIV viral load (HIV RNA was measured by Roche TaqMan 2.0,the lower limit of quantification is 20 copies per milliliter). Furthermore, the CSF sample was collected for routine CSF parameters and immunological analysis at all visits. The information of imaging and cognitive function information collected was collected at screening and week $8 \otimes 24$. (Table S1)

\section{Clinical Effectiveness and safety evaluation}

The primary endpoint of this pilot cohort study wasthe effectiveness of lenalidomide therapy which was determined by the clinical manifestations of participants, routine CSF parameters, and radiographic response within 24 weeks post-treatment. Routine CSF parameters including cell count, protein, glucose, were measured at each visit.Radiographic response forlenalidomide therapy was assessedbymagnetic resonance imaging (MRI) of the brain.Presenting clinical, CSF routine CSF parameters and imaging information were compared between the baseline and post-treatment. Drug safety is mainly evaluated 
through adverse events, vital signs, and laboratory parameters.At each visit, vital signs, height,and weight were assessed and laboratory tests offull blood counts, biochemistry, coagulation function, andCD $4^{+} \mathrm{T}-$ lymphocytesand other immune cell subsetscount were done. Demographic data (age, gender), medical history, laboratory parameters, and imaging finding were obtained from medical records or hospital databases.

\section{Detection of CSF inflammatory cytokines}

$\mathrm{CSF}$, collected from participants, were immediately centrifuged and the supernatant was stored at $-80^{\circ} \mathrm{C}$ until the test.Cytokineconcentration was determined bya magnetic bead-based multiplex panel (Luminex》

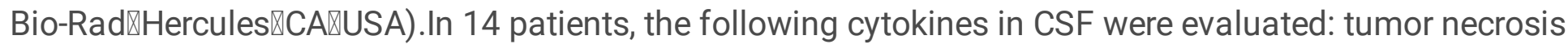
factor-a (TNF- $a$ ), interferon- $\gamma$ (IFN- $\gamma$ ), interleukin (IL)-1a, IL-1 $\beta$, IL-2, IL-3, IL-4, IL-6, IL-8, IL-10, IL-12p40, IL12p70, IL-13, IL-17A, interferon-gamma induced protein 10 (IP-10), monocyte chemotactic protein-1 (MCP1), macrophage inflammatory protein (MIP)-1a, MIP-1 $\beta$, granulocyte colony-stimulating factor (G-CSF), granulocyte-macrophage colony-stimulating factor (GM-CSF), soluble CD40 ligand (sCD40L),Complement $\mathrm{C} 3$,complement Factor $\mathrm{H}$ (CFH), fibroblast growth factor -2 (FGF-2), platelet-derived growth factor$A A(P D G F-A A)$,growth-related oncogene (GRO), alpha 2-macroglobulin (a2M), apolipoprotein Al (Apo.Al), apolipoprotein E (Apo. E), amyloid beta 1-42(Aß42), total tau (tTau),phosphorylated tau(pTau). The concentrations of Cytokines were expressed in $\mathrm{pg} / \mathrm{ml}$ and determined pre- and post-lenalidomide therapy.Please refer to Table $S 2$ for the explanation of abbreviations

\section{Statistical analysis}

Demographic and laboratory data are expressed as median (interquartile range (IQR)) for continuous variables.Categorical variables weredescribedas $\mathrm{n}(\%)$.Efficacyand safety endpoints are reported based on the full analysis set which consists of all patients receiving at least one dose of study treatment with assessable data. All clinical and imaging data items were recorded in Microsoft Office Excel (version 2019). And Wilcoxon matched-pairs signed-rank tests were applied to compare baseline and post-therapy values. A two-sided $p$ value less than 0.05 was considered statistically significant and $p$ values were adjusted for multiple comparisons via segmentation method.And all statistical analyses were performed with SPSS (version 23). Each parameter was tested for normal distribution with SPSS software.Gene ontology (GO) analysis was performed using the GO consortium web tool(www.geneontology.org). Furthermore, graphical charts were generated in GraphPad Prism (version 8). This study is registered with China Clinical Trial Registry website (www.chictr.org/cn/, Number: ChiCTR1900023184)

\section{Results}

\section{Participant characteristics}


Between Jan2017 and Oct2020, we screened99 patients with successful induction therapy for HIV-CM, 72 of whom had developed central nervous system inflammation post-ART. And we finally identified 14 eligible patients on the basis of our inclusion and exclusion criteria (Figure 1). Of 14 patients enrolled, 11 completed the 24-week follow-up, and 3 participants withdrew for personal reasons (relocation;poor economy) (Figure 1). All the participants are Asian men, witha median age 32.50 (IQR 30.50-39.25) years. Atbaseline, the median time from CM to ART initiation was 25.50 (IQR 18.50-36.50). And the mediantime from ART initiation to Screening was 558.00 (IQR 314.00-882.25『days. The median CD 4 ${ }^{+}$-lymphocyte count was 151.50 (117.00-342.25)/ul and 11 participants' plasma HIV RNA is below the detection limit. The predominant clinical manifestations were fever $₫ 4,28.6 \% \bigotimes$, headache, $₫ 7,50 \% \otimes$ Cognitive impairment $\llbracket 4,28.6 \% \bigotimes$, and seizures $\varangle 2,14.3 \% \bigotimes($ Table1,Table S3)

\section{Rapid clinical remission followed lenalidomide therapy}

All participantsreceived at least one cycle of lenalidomide. When all participants completed the first cycle of lenalidomide therapy (week 4), clinical presentations such as fever, headache, and convulsions were fully recovered. Only 1 (7.1\%) patient still showed Cognitive impairment, but get recovery at week 12 (Table 2).

Table 2 Rapid clinical remission of participants followed lenalidomide therapy

\begin{tabular}{|llllll|}
\hline Time & Week 0 & Week4 & Week 8 & Week 12 & Week 24 \\
\hline Fever $\mathrm{n}(\%)$ & $4(28.6 \rrbracket$ & $0(0.0)$ & $0(0.0)$ & $0(0.0)$ & $0(0.0)$ \\
\hline Headache $\mathrm{n}(\%)$ & $7(50.0)$ & $0(0.0)$ & $0(0.0)$ & $0(0.0)$ & $0(0.0)$ \\
\hline Cognitiveimpairment $\mathrm{n}(\%)$ & $4(28.6)$ & $1(7.1)$ & $1(7.1)$ & $0(0.0)$ & $0(0.0)$ \\
\hline Seizures $\mathrm{n}(\%)$ & $2(14.3)$ & $0(0.0)$ & $0(0.0)$ & $0(0.0)$ & $0(0.0)$ \\
\hline
\end{tabular}

\section{Rapid reduction inroutine CSF parameters followed lenalidomide therapy}

Furthermore, we observed a significant reduction inroutine CSF parameters frombaseline (Figure 2). The medianbaseline CSFWBC countwas $35 \times 10^{6} / \mathrm{L}(\mathrm{IQR} 4.50$ to 90.00$)$. Significantreductionoccurredat week 4 [Median count 3.00 (IQR 0-45.00 P=0.009)] from baseline. At the same time, the CSF protein also decreases significantly in a short time. MedianCSF protein concentration decreases from $1.39 \mathrm{~g} / \mathrm{L}$ (IQR 0.74-3.23) at baseline to 0.91 g/L (IQR 0.63-1.41) at week 4 ( $P=0.004)$. Additionally, CSF WBC count, CSF proteinremainedstable and approached the normal range through week 24 [WBC count $10.00 \times 10^{6} / \mathrm{L}$ (IQR2.00-14.00),protein 0.75g/L (IQR 0.52-1.08)]. Atbaseline,median CSF albumin and immunoglobin G (IgG) were 79.20 (48.40-149.75) and 13.10 (7.26-160.50). Median CSF albumin decrease from 79.20 $\mathrm{mg} / \mathrm{L}$ (IQR 48.40-149.75) at baseline to 42.35 (IQR 32.68-54.40) at week 24 ( $\mathrm{P}=0.017)$. And after the 24- 
week lenalidomide therapy, CSF IgG levels were also decreased, though not statistically significant. There was no significant change in cerebrospinal fluid pressure and chlorine at each visit point,while CSF glucose level was slightly elevated $(0.195 \% \mathrm{Cl} 0$ to $0.75 \mathrm{P}=0.044)$ at week 24 from baseline (Table S4).

\section{Rapid reduction in imaging characteristics followed lenalidomide therapy}

The baseline imaging characteristics of all participants are summarized inTable 3.it was observed that brain lesions mainly located in the meninges $(10,71.4 \%)$, Cerebral white matter $(5,35.7 \%)$ and basal ganglia (4, 28.6\%), and brain parenchyma (3, 21.4\%). Among the participants, $10(71.4 \%)$ patients showed inflammatory lesions, and other form of lesions included ischemia, encephalomalacia, and hemorrhage. In addition, it is worth noting that there are $3(28.6 \%)$ patients with an 2unidentified lesion. An obvious remission in the inflammatorylesionwas observed in $8(8 / 10,80 \%)$ of patients postlenalidomide therapy. And additional ischemic injury reduction was present in $3(3 / 4,75 \%)$ of participants. Furthermore,there is $1(1 / 1100 \%)$ patient had hemorrhagic lesion absorbed.

Table 3 Imaging characteristics of participants

\begin{tabular}{|lll|}
\hline Cranial MRI & Baseline $\mathbf{n}(\%)$ & 24W n (\%)* \\
\hline Lesion Location & & \\
\hline Meninges & $10(71.4)$ & $2(14.3)$ \\
\hline Brain parenchyma & $3(21.4)$ & $1(7.1)$ \\
\hline Basal ganglia & $4(28.6)$ & $3(21.4)$ \\
\hline Lesion nature & & \\
\hline Inflammation & $10(71.4)$ & $2(14.3)$ \\
\hline Encephalomalacia & $2(14.3)$ & $2(14.3)$ \\
\hline Hemorrhagic lesion & $1(7.1)$ & $0(0)$ \\
\hline Cerebral ischemia & $4(28.6)$ & $1(7.1)$ \\
\hline Unidentifiedlesion & $3(21.4)$ & $3(21.4)$ \\
\hline
\end{tabular}

*The data show that number of lesions, which is not getting better after 24 weeks of treatment with lenalidomide 


\section{Significant decrease in proinflammatory Th1 profile associated cytokine}

For the exploratory analysis, we tryto look for the most profound changes in the concentrations of CSF cytokines. Patients had high levels of proinflammatory Th1 profile such as TNF-a, G-CSF, IL-6, and so on at thetimeof enrollment. Themedian baseline TNF-a was $18.86 \mathrm{pg} / \mathrm{ml}$ (IQR4.50-27.17). TNF-a showed a significant decrease at week $4(8.09 \mathrm{pg} / \mathrm{mgIQR} 4.06-20.51 \mathrm{P}=0.005)$ compared with the baseline, and its concentration tends to decrease within 24 weeks (Figure 3 ). In addition, it was observed that cerebrospinal fluid G-CSF and IL-6 were at a high level, the median baseline G-CSF and IL-6 were 83.72 $\mathrm{pg} / \mathrm{ml}$ (IQR 3.90-562.21) and $191.37 \mathrm{pg} / \mathrm{ml}$ (IQR 3.90-3958.00), respectively. Compared to the baseline,the median G-CSF decreased by $90.13 \%$ at week $4(8.26 \mathrm{pg} / \mathrm{ml}$ IQR 3.7-16.25 P=0.005) and decreased by $96.54 \%$ at week $24(3.20 \mathrm{pg} / \mathrm{ml}$ IQR 2.83-3.90 P=0.008). IL-6 decreases occurred at week 4 frombaseline and remained reduced at week $24(2.89 \mathrm{pg} / \mathrm{ml}$ IQR 2.84-14.65 P=0.008) (Figure 3). In addition, it was observed that IL-17A, IL-10, a2M, Apo.Al, CFH, and Complement C3 decreased significantly during the 24week follow-up (Figure S1). The GO pathway analysis results indicated that thesecytokineswereinvolvedintheregulationofinflammatoryresponseandregulationof the immune effector process(Figure S2).

\section{Safety analysis of lenalidomide}

Safety analysis was done in 14 patients who had received at least one dose of lenalidomide. 2 (14.2\%) patients had a mild skin rash,which resolved spontaneously withoutdruginterruption.No study drugrelated serious adverse events were reported, no participant died during the study.

No patient withdrew from therapy because of unacceptable toxicity. And, it was showninourstudythat lenalidomide had a modest inhibitory effect on the bone marrow and has a greater impact on neutrophils. Comparedtothebaseline, the patient's white blood cells, neutrophils, and platelets were slightly reduced during the follow-up visit. The median white blood cells and platelets count dropped to the lowest value

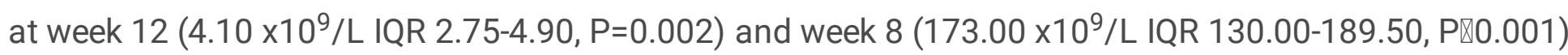
respectively, which remained within the normal range. Furthermore, there was a significant decrease in neutrophils count, which reached a minimum at week 12 ( $1.10 \times 10^{9} / \mathrm{L}$ IQR 0.93-1.65, P=0.002). However, the levels of the aforementioned indices at week 24 were elevated to a certain extent and close to normal levels (Table S5).In addition, except forblood urea nitrogen and D-dimer, there was no obvious change in liver and kidney functions, blood lipids, and coagulationfunctions duringthe follow-up period. There was a slight increase in blood urine nitrogen and total bilirubin atweek 24 but still within the normal range. And there were significantly fewer D-dimer at week 24 relative to the baseline. We also analyzed the CD $4^{+} T$ lymphocytes and $\mathrm{CD}^{+}{ }^{+}$-lymphocytes counts and found no significant differences following lenalidomide therapy.

\section{Discussion}


Inthis trial we aim to test the concept: lenalidomide, as an immunomodulator, could be considered as an alternative therapy for persistent intracranial inflammation in HIV-CM Patients.And in this study, it was observed that rapid clinical remission followed lenalidomide therapy. within 3 weeks after lenalidomide treatment, symptoms of meningitis syndrome disappeared in most patients. Inflammatory indices of CSF such as WBC count, protein, and albumin also reduced significantly and gradually approached the normal range.Changes in radiological imaging were also observed among these patients, two of whom had an obvious remission in the inflammatory lesion post lenalidomide therapy. And other patients also showed different degrees of imaging improvement.In addition to observing rapid clinical remission followed lenalidomide therapy, we also found significant reductions in Th1 associated pro-inflammatory cytokines such as TNF-a, IL- 6 and G-CSF in the cerebrospinal fluid. And the GO pathway analysis results indicated that thesecytokineswereinvolvedintheregulationofinflammatoryresponseandregulationofimmune effector process.

In the trial, patients enrolled were those HIV-1 infected individuals with cryptococcal meningitis. Receiving standard antifungal and antiviral therapy, these patients had negative cerebrospinal fluid cryptococcal cultures and plasma viral loads tended to be stable. However, the cerebrospinal fluid indicators continued to be abnormal, and the clinical manifestations did not improve significantly, or even worsened. It was believed that the occurrence of the above phenomenon is mediated by chronic intracranial inflammation, which might be a cause of immune reconstitution inflammatory syndrome(IRIS) or HIV-associated neurocognitive diseases (HAND) [9]. And itwasobservedinourstudy thatparticipants hadahighlevelof Th1associated cytokine TNF- $a$ and the pro-inflammatory cytokines G-CSF, IL-6, IL-17A at baseline, which is consistent with previously reported in the literature[13,23].The consequences of the exaggerated Th1 bias response are persistent malignant inflammation, blood-brain barrier, tissue damage, and increased intracranial pressure, which eventually lead to cognitive dysfunction and even death[24].

Recently, it is believed that anti-inflammatory and neuron protection are particularly important for chronic CNS inflammation. In the past, corticosteroids have been tried to treat persistent intracranial inflammation[25]. It was reported in CM-IRIS without HIV infection patients that after using corticosteroids headache was immediately relieved and the pressure of the CSF went downwithin a short time [26]. And corticosteroids proved to be equally effective in the HIV infected individual with CM-IRIS in some literature[27, 28]. However, cases of corticosteroid-dependent were always reported, where inflammation relapses usually occurred when corticosteroids were tapered or interrupted[29]. Additionally, corticosteroid use in immunocompromised patients may potentially increase the risk of infection and mortality[30]. Furthermore, other immunomodulators have been tested in severe cases of chronic inflammation that mediates brain injury.In HIV-infected patients with corticosteroid-dependent and lifethreatening CM-IRIS,thalidomide administration induced rapid clinical remission[29]. However, the duration of thalidomide use in these cases was generally long which all more than a year. Also, it is worth noting that no definite immunomodulatory effect of thalidomide on cytokine levels was observed by the researcher. In addition, the side effects of thalidomide are also worrying, in this study, two of the three experienced adverse reactions, although only mild[29]. A randomized trial of thalidomide in children with tuberculous meningitis was terminated prematurely due to severe adverse events in the thalidomide 
arm[31]. In addition, it is observed that in ART-naïve adult HIV patients, thalidomide caused an intense increase in T cell activation and inflammation, which further led to a decrease in CD $4^{+}$T-lymphocytes count[32]. In addition, anti-inflammatory strategies such as minocycline, selegiline, and so on have been tested in several clinical trials, but none of these exerted a significant clinically beneficial effect [33]. A recent study, however, has demonstrated that intranasal delivered insulin could reverse neuronal injury,whichmay be related to the reduction of microglia activation and the suppression of chronic inflammation, and is now being tested in PLWH[34, 35].

Compared to previous studies, the drug used in this study is lenalidomide, an analog of thalidomide, which is thought to have more powerful immunomodulatory functions but fewer side effects than thalidomide[36]. Different from previous reports of thalidomide [29], In this trial, the treatment cycle of lenalidomide was only a half year and it is intermittent. In addition to observing rapid clinical remission followed lenalidomide therapy, we also found significant reductions in pro-inflammatory cytokines such as TNF-a, IL-6, and G-CSF in the cerebrospinal fluid. Thus, thereisreasontobelievethat lenalidomide may effectively inhibit the intense Th1 pro-inflammatory response in the brain, thereby improving clinical symptoms. Furthermore, compared to corticosteroids and thalidomide,lenalidomide seems to be safe and well-tolerated[12]. In our study, there were no severe adverse events reported during the follow-up period, and only 2 patients had a mild skin rash. In addition, compared to baseline, no major changes in blood routine, liver, and kidney function indicators were noted during follow-up. In addition, unlike thalidomide, compared to the baseline, CD $4^{+}$T-lymphocytes cells increased at 24 weeks, although the difference was not statistically significant.

However, some limitations need to be recognized. First, this study was limited by the small sample size and short duration of follow-up.Considering that lenalidomide has never been tested in HIV-CM populations so far, we only launched a pilot cohort of 14 patients. Second, this was a single-arm prospective study without a control group, which limited the ability to determine the effects of interventions on clinical outcomes. Third, the population enrolled in our study were mainly middle-aged Chinese men with HIV-CM, which prevented the extrapolation of our findings to other populations. Fourth, for the lack of effective quantitative tools, the information of MRI has not been fully displayed, though imaging findings of most patients during the follow-up were accessible. And we plan to recruit a second international multicenter randomized controlled trial to verify the results of our study when initial success was achieved.

\section{Conclusions}

This study demonstrated that lenalidomide is safe, well-tolerated, and can achieve dramatic clinical improvement in HIV-CM patients who develop persistent intracranial inflammation.Furthermore, itexerts an immunomodulatory effect on CSF inflammatory cytokines. Given the close association between cytokines and neuroinflammation[12],lenalidomide could beconsidered as an alternative therapeutic strategy in HIV-CM patients with persistent intracranial inflammation. Further studies should be carried 
out regarding the clinical application and specific mechanism of lenalidomide in HIV-CM patients with persistent intracranial inflammation.

\section{Abbreviations}




\begin{tabular}{|c|c|}
\hline $\mathrm{a} 2 \mathrm{M}$ & alpha 2-macroglobulin \\
\hline Apo.Al & apolipoprotein Al \\
\hline Apo. E & apolipoprotein E \\
\hline ART & antiretroviral therapy \\
\hline Aß42 & amyloid beta 1-42 \\
\hline $\mathrm{CFH}$ & complement Factor $\mathrm{H}$ \\
\hline $\mathrm{CM}$ & cryptococcal meningitis \\
\hline CNS & central nervous system \\
\hline CSF & cerebrospinal fluid \\
\hline FGF-2 & fibroblast growth factor -2 \\
\hline G-CSF & granulocyte colony-stimulating factor \\
\hline GM-CSF & granulocyte-macrophage colony-stimulating factor \\
\hline GO & gene ontology \\
\hline GRO & growth-related oncogene \\
\hline HIV & human immunodeficiency \\
\hline HIV-CM & HIV-associated cryptococcal meningitis \\
\hline IFN-Y & interferon-y \\
\hline $\lg G$ & immunoglobin $\mathrm{G}$ \\
\hline IL-1a & interleukin-1a \\
\hline IL-1 $\beta$ & interleukin-1 $\beta$ \\
\hline IL-10 & interleukin-10 \\
\hline IL-12p40 & interleukin-12p40 \\
\hline IL-12p70 & interleukin-12p70 \\
\hline INSTI & Integrase strand transfer inhibitors \\
\hline IP-10 & interferon gamma induced protein 10 \\
\hline IQR & interquartile range \\
\hline MCP-1 & monocyte chemotactic protein 1 \\
\hline MIP-1a & macrophage inflammatory protein-1a \\
\hline MIP-1 $\beta$ & macrophage inflammatory protein- $1 \beta$ \\
\hline
\end{tabular}

Page 12/21 


\begin{tabular}{|ll|} 
MRI & magnetic resonance imaging \\
\hline NNRTI & nonnucleoside reverse transcriptase inhibitors \\
\hline PDGF-AA & platelet-derived growth factor-AA \\
PI & protease inhibitors \\
PLWH & people living with HIV \\
pTau & phosphorylated tau \\
\hline sCD40L & soluble CD40 ligand \\
\hline Th1 & T helper1 \\
tTau & total tau \\
\hline TNF-a & tumor necrosis factor-a \\
WBC & white blood cell \\
\hline
\end{tabular}

\section{Declarations}

\section{Ethics approval and consent to participate}

The trial protocol and other documents were approved by the Institutional Ethics Committee in First Affiliated Hospital of Zhejiang University in accordance with the Helsinki Declaration. Informed consent was obtained from all patients in the trial.

\section{Consent for publication}

All authors have approved this manuscript.

\section{Availability of data and materials}

The datasets supporting the conclusions of this article are available from the correspondingauthor on reasonable request.

\section{Competing interests}

The authors declare that they have no competing interests.

\section{Funding}




\section{Authors' contributions}

$B Z$ contributed to the conception and design of the study. All authors (BZ, RT, ZKW, $J J H, X L, X R P, Y Z G, X L Z, Y H)$ contributed to the acquisition and analysis of data. RT,ZKW,JJH contributed to drafting the text, preparing the tables and figures and they contribute equally to the article. All authors read and approved the final manuscript

\section{Acknowledgments}

Not applicable

\section{References}

1. Bowen LN, Smith B, Reich D, Quezado M, Nath A: HIV-associated opportunistic CNS infections: pathophysiology, diagnosis and treatment.Nat Rev Neurol 2016, 12:662-674.

2. Elsegeiny W, Marr KA, Williamson PR: Immunology of Cryptococcal Infections: Developing a Rational Approach to Patient Therapy.Front Immunol 2018, 9:651.

3. Haddow LJ, Colebunders R, Meintjes G, Lawn SD, Elliott JH, Manabe YC, Bohjanen PR, Sungkanuparph S, Easterbrook PJ, French MA, Boulware DR: Cryptococcal immune reconstitution inflammatory syndrome in HIV-1-infected individuals: proposed clinical case definitions. Lancet Infect Dis 2010, 10:791-802.

4. Rajasingham R, Smith RM, Park BJ, Jarvis JN, Govender NP, Chiller TM, Denning DW, Loyse A, Boulware DR: Global burden of disease of HIV-associated cryptococcal meningitis: an updated analysis.Lancet Infect Dis 2017, 17:873-881.

5. Anjum S, Williamson PR: Clinical Aspects of Immune Damage in Cryptococcosis. Curr Fungal Infect Rep 2019, 13:99-108.

6. Nunnari G, Gussio M, Pinzone MR, Martellotta F, Cosentino S, Cacopardo B, Celesia BM: Cryptococcal meningitis in an HIV-1-infected person: relapses or IRIS? Case report and review of the literature.Eur Rev Med Pharmacol Sci 2013, 17:1555-1559.

7. Su T, Wit FW, Caan MW, Schouten J, Prins M, Geurtsen GJ, Cole JH, Sharp DJ, Richard E, Reneman L, et al: White matter hyperintensities in relation to cognition in HIV-infected men with sustained suppressed viral load on combination antiretroviral therapy.Aids 2016, 30:2329-2339.

8. Yang Y, Li M, Yang L, Tian Q, Qin B: Clinical, radiographic features and long-term outcomes of paradoxical cryptococcosis-associated immune reconstitution inflammatory syndrome secondary to the ventriculoperitoneal shunt.J Infect 2021, 83:607-635. 
9. McArthur JC, Johnson TP: Chronic inflammation mediates brain injury in HIV infection: relevance for cure strategies.Curr Opin Neurol 2020, 33:397-404.

10. Spudich S, Robertson KR, Bosch RJ, Gandhi RT, Cyktor JC, Mar H, Macatangay BJ, Lalama CM, Rinaldo C, Collier AC, et al: Persistent HIV-infected cells in cerebrospinal fluid are associated with poorer neurocognitive performance.J Clin Invest 2019, 129:3339-3346.

11. Lindl KA, Marks DR, Kolson DL, Jordan-Sciutto KL: HIV-associated neurocognitive disorder: pathogenesis and therapeutic opportunities.J Neuroimmune Pharmacol 2010, 5:294-309.

12. Balasko A, Keynan Y: Shedding light on IRIS: from Pathophysiology to Treatment of Cryptococcal Meningitis and Immune Reconstitution Inflammatory Syndrome in HIV-Infected Individuals. HIV Med 2019, 20:1-10.

13. Boulware DR, Bonham SC, Meya DB, Wiesner DL, Park GS, Kambugu A, Janoff EN, Bohjanen PR: Paucity of initial cerebrospinal fluid inflammation in cryptococcal meningitis is associated with subsequent immune reconstitution inflammatory syndrome.J Infect Dis 2010, 202:962-970.

14. Saylor D, Dickens AM, Sacktor N, Haughey N, Slusher B, Pletnikov M, Mankowski JL, Brown A, Volsky DJ, McArthur JC: HIV-associated neurocognitive disorder-pathogenesis and prospects for treatment.Nat Rev Neurol 2016, 12:234-248.

15. BrienzeVMS, André JC, Liso E, Louis IV: Cryptococcal Immune Reconstitution Inflammatory Syndrome: From Blood and Cerebrospinal Fluid Biomarkers to Treatment Approaches.Life (Basel) 2021, 11.

16. Brust JCM, McGowan JP, Fine SM, Merrick ST, Radix AE, Vail RM, Stevens LC, Hoffmann CJ, Gonzalez CJ. Management of Immune Reconstitution Inflammatory Syndrome (IRIS) [Internet]. Baltimore (MD): Johns Hopkins University; 2021, 4.

17. Li J, Huang XF, Cai QQ, Wang C, Cai H, Zhao H, Zhang L, Cao XX, Gale RP, Zhou DB: A prospective phase II study of low dose lenalidomide plus dexamethasone in patients with newly diagnosed polyneuropathy, organomegaly, endocrinopathy, monoclonal gammopathy, and skin changes (POEMS) syndrome.Am J Hematol 2018, 93:803-809.

18. Salles G, Duell J, González Barca E, Tournilhac O, Jurczak W, Liberati AM, Nagy Z, Obr A, Gaidano G, André $M$, et al: Tafasitamab plus lenalidomide in relapsed or refractory diffuse large B-cell lymphoma (L-MIND): a multicentre, prospective, single-arm, phase 2 study.Lancet Oncol 2020, 21:978-988.

19. Raza S, Safyan RA, Lentzsch S: Immunomodulatory Drugs (IMiDs) in Multiple Myeloma.Curr Cancer Drug Targets 2017, 17:846-857.

20. Tsenova L, Mangaliso B, Muller G, Chen Y, Freedman VH, Stirling D, Kaplan G: Use of IMiD3, a thalidomide analog, as an adjunct to therapy for experimental tuberculous meningitis.Antimicrob Agents Chemother 2002, 46:1887-1895.

21. Anwer S, Collings F, Trace K, Sun Y, Sternberg A: Cerebrospinal fluid penetrance of lenalidomide in meningeal myeloma.Br J Haematol 2013, 162:281-282.

22. Calimeri T, Steffanoni S, Gagliardi F, Chiara A, Ferreri AJM: How we treat primary central nervous system lymphoma.ESMO Open 2021, 6:100213. 
23. Hong S, Banks WA: Role of the immune system in HIV-associated neuroinflammation and neurocognitive implications.Brain Behav Immun 2015, 45:1-12.

24. Albarillo F, O'Keefe P: Opportunistic Neurologic Infections in Patients with Acquired Immunodeficiency Syndrome (AIDS).Curr Neurol Neurosci Rep 2016, 16:10.

25. Perfect JR, Dismukes WE, Dromer F, Goldman DL, Graybill JR, Hamill RJ, Harrison TS, Larsen RA, Lortholary $\mathrm{O}$, Nguyen $\mathrm{MH}$, et al: Clinical practice guidelines for the management of cryptococcal disease: 2010 update by the infectious diseases society of america. Clin Infect Dis 2010, 50:291-322.

26. Wu G, Guo X, Wang Y, Hu Z: Clinical and Radiographic Features of Cryptococcal Neoformans Meningitis-associated Immune Reconstitution Inflammatory Syndrome.Sci Rep 2020, 10:9948.

27. Skiest DJ, Hester LJ, Hardy RD: Cryptococcal immune reconstitution inflammatory syndrome: report of four cases in three patients and review of the literature.J Infect 2005, 51:e289-297.

28. Maciel RA, Ferreira LS, Wirth F, Rosa PD, Aves M, Turra E, Goldani LZ: Corticosteroids for the management of severe intracranial hypertension in meningoencephalitis caused by Cryptococcus gattii: A case report and review.J Mycol Med 2017, 27:109-112.

29. Brunel AS, Reynes J, Tuaillon E, Rubbo PA, Lortholary O, Montes B, Le Moing V, Makinson A: Thalidomide for steroid-dependent immune reconstitution inflammatory syndromes during AIDS.Aids 2012, 26:2110-2112.

30. Day J, Imran D, Ganiem AR, Tjahjani N, Wahyuningsih R, Adawiyah R, Dance D, Mayxay M, Newton P, Phetsouvanh R, et al: CryptoDex: a randomised, double-blind, placebo-controlled phase III trial of adjunctive dexamethasone in HIV-infected adults with cryptococcal meningitis: study protocol for a randomised control trial.Trials 2014, 15:441.

31. Schoeman JF, Springer P, van Rensburg AJ, Swanevelder S, Hanekom WA, Haslett PA, Kaplan G: Adjunctive thalidomide therapy for childhood tuberculous meningitis: results of a randomized study.J Child Neurol 2004, 19:250-257.

32. Vergara TRC, Samer S, Santos-Oliveira JR, Giron LB, Arif MS, Silva-Freitas ML, Cherman LA, Treitsman MS, Chebabo A, Sucupira MCA, et al: Thalidomide is Associated With Increased T Cell Activation and Inflammation in Antiretroviral-naive HIV-infected Individuals in a Randomised Clinical Trial of Efficacy and Safety.EBioMedicine 2017, 23:59-67.

33. Eggers C, Arendt G, Hahn K, Husstedt IW, Maschke M, Neuen-Jacob E, Obermann M, Rosenkranz T, Schielke E, Straube E: HIV-1-associated neurocognitive disorder: epidemiology, pathogenesis, diagnosis, and treatment.J Neurol 2017, 264:1715-1727.

34. Kim BH, Kelschenbach J, Borjabad A, Hadas E, He H, Potash MJ, Nedelcovych MT, Rais R, Haughey $\mathrm{NJ}$, McArthur JC, et al: Intranasal insulin therapy reverses hippocampal dendritic injury and cognitive impairment in a model of HIV-associated neurocognitive disorders in EcoHIV-infected mice.Aids 2019, 33:973-984.

35. Mamik MK, Asahchop EL, Chan WF, Zhu Y, Branton WG, McKenzie BA, Cohen EA, Power C: Insulin Treatment Prevents Neuroinflammation and Neuronal Injury with Restored Neurobehavioral Function in Models of HIV/AIDS Neurodegeneration.J Neurosci 2016, 36:10683-10695. 
36. Kumar N, Sharma U, Singh C, Singh B: Thalidomide: chemistry, therapeutic potential and oxidative stress induced teratogenicity.Curr Top Med Chem 2012, 12:1436-1455.

\section{Tables}

\section{Table1 Participant characteristics}




\begin{tabular}{|c|c|}
\hline \multicolumn{2}{|l|}{ Characteristics } \\
\hline Agelyear[ & $32.50(30.50-39.25)$ \\
\hline \multicolumn{2}{|l|}{ sex-no.(\%) } \\
\hline Male & $14(100)$ \\
\hline Female & $0(0)$ \\
\hline \multicolumn{2}{|l|}{ Race or ethnic group-no.(\%) } \\
\hline Asian & $14(100)$ \\
\hline Other race & $0(0)$ \\
\hline BMI & $23.06(20.19-26.43)$ \\
\hline Time from CM to ART initiation, $d$ & $25.50(18.50-36.50)$ \\
\hline Time from ART initiation to Screening, $d$ & $558.00(314.00-882.25 \rrbracket$ \\
\hline \multicolumn{2}{|l|}{ ART regimen-no.]\% } \\
\hline 2NRTI+1NNRTI & $2(14.3 \rrbracket$ \\
\hline 2NRTI+1PI & $1(7.1 \otimes$ \\
\hline 2NRTI+1 INSTI & $11(78.6 \rrbracket$ \\
\hline CD4+ T-lymphocytes count /ul & $151.50(117.00-342.25)$ \\
\hline \multicolumn{2}{|l|}{ HIV-RNA-no.]\%ם } \\
\hline$₫ 50$ copies $/ \mathrm{ml}$ & $11(78.6)$ \\
\hline 50-200 copies/ml & 0 \\
\hline$\bigotimes 200$ copies $/ \mathrm{ml}$ & $3(22.4)$ \\
\hline \multicolumn{2}{|l|}{ CD4+ T-lymphocytescount(Screen)-no.(\%) } \\
\hline$<200$ cells/ul & $9(64.3 \rrbracket$ \\
\hline 200-500 cells/ul & $5(35.7 \rrbracket$ \\
\hline$>500$ cells/ul & $0(0)$ \\
\hline \multicolumn{2}{|l|}{ clinical presentation-no.(\%) } \\
\hline Fever & $4(28.6 \rrbracket$ \\
\hline Headache & $7(50)$ \\
\hline Cognitive impairment & $4(28.6)$ \\
\hline Seizures & $2(14.3)$ \\
\hline
\end{tabular}


Data are median (IQR) or n (\%)

\section{Figures}

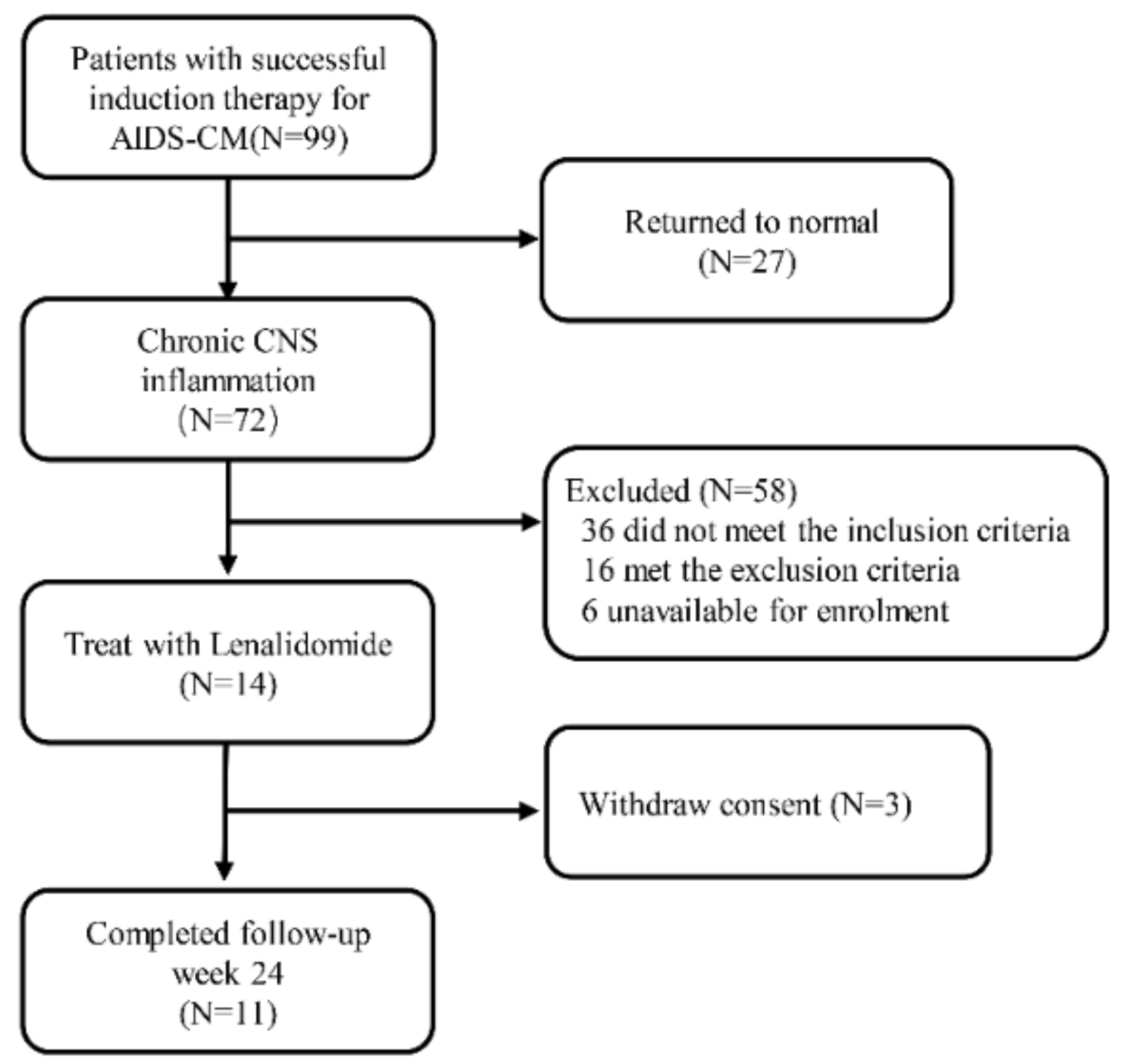

Figure 1

Trial profile 

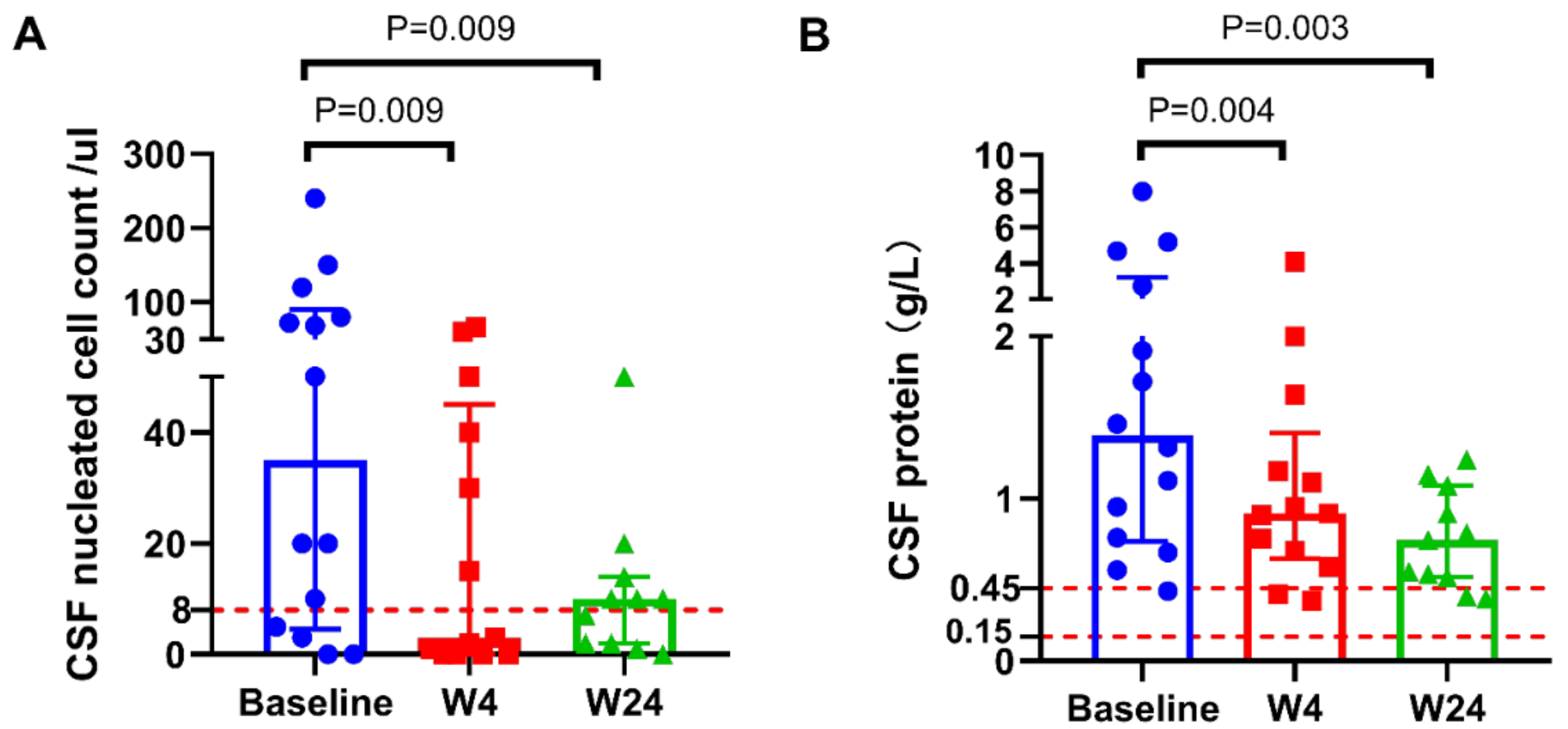

Figure 2

CSF parameters change during 24 weeks followup Rapid reduction in CSF WBC and protein was observed followed lenalidomide therapy. Red dotted lines represent the range of normal reference values for CSF WBC count and CSF protein levels.W: Week,W4: Week 4,W24:Week 24.
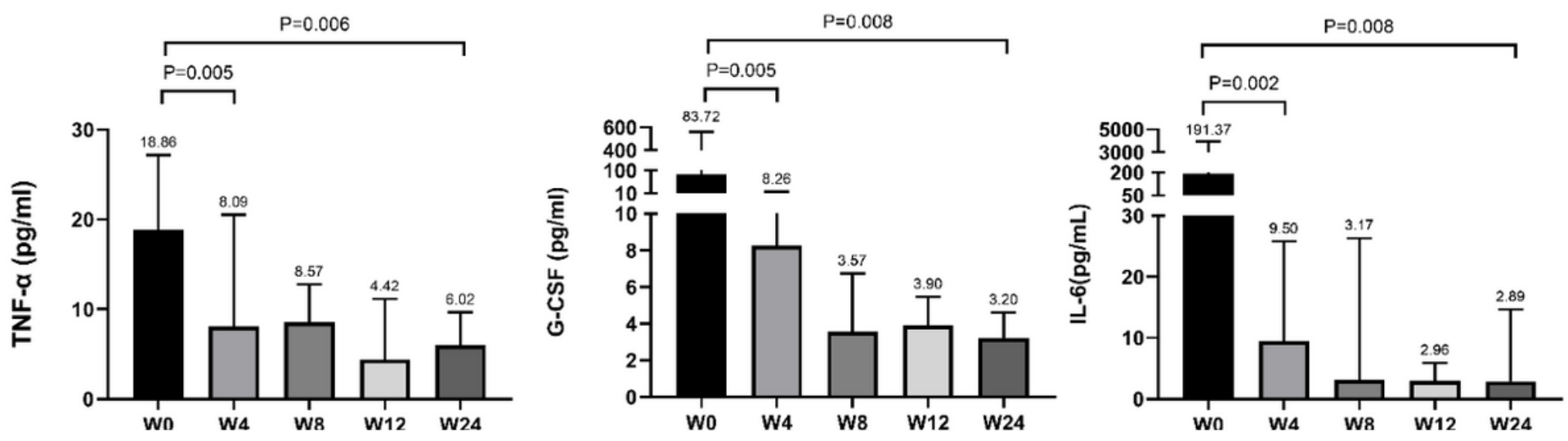

Figure 3

CSF Th1 associated inflammatory cytokines measurements Concentrations of TNF-a, G-CSFand IL-6 in the CSF from patients treated with lenalidomidede crease significantly during follow-up. W: week; TNF-a: tumor necrosis factor-a; G-CSF: granulocyte colony-stimulating factor; IL-6:interleukin-6

\section{Supplementary Files}

This is a list of supplementary files associated with this preprint. Click to download. 
- SupplementaryMaterial.docx

Page 21/21 